\title{
Influencia de la intensidad de uso sobre algunas propiedades físicas en un suelo del Valle del Cauca, Colombia
}

\section{Influence of the intensity of use on some physical properties in a soil of the Valle del Cauca, Colombia} Influência da intensidade do uso, sobre algumas propriedades físicas em um solo de Valle del Cauca, Colômbia

\author{
Alveiro Salamanca Jiménez ${ }^{1}$ \& Edgar Amézquita Collazos² \\ 1Ingeniero Agrónomo, Magister y Doctor en Suelos y Biogeoquímica. ²Ingeniero Agrónomo, \\ Magister en Física y Conservación de Suelos, Doctor en Física de Suelos \\ 'Disciplina de Suelos, Centro Nacional de Investigaciones de Café -Cenicafé. Chinchiná, Caldas. \\ Colombia. ${ }^{2}$ Investigador Independiente. Cali. Colombia. \\ 1alveiro.salamanca@cafedecolombia.com, ${ }^{2}$ e.amezquitac@gmail.com
}

\section{Resumen}

La intensidad de uso y manejo del suelo generalmente altera el comportamiento de sus propiedades físicas y acelera su degradación. Con el fin de medir el impacto de dicha intensidad, se llevó a cabo un muestreo en la estación experimental del CIAT-Palmira, Valle del Cauca, Colombia. Se escogieron seis lotes con diferentes historiales, desde uso semestral muy intensivo hasta uso reducido con descanso por más de 10 años, y se tomaron muestras de suelo a cinco profundidades del perfil para evaluar las siguientes propiedades: conductividad hidráulica, permeabilidad al aire, porosidad total, porosidad drenable, compactabilidad, porosidad residual, materia orgánica y resistencia mecánica a la penetración. Se encontró que el uso de maquinaria y las labores de fangueo mejoraron las propiedades físicas sólo en los $15 \mathrm{~cm}$ superficiales, pero han causado graves problemas de compactación en las profundidades inferiores. El pisoteo del ganado también ha compactado el perfil, limitando las condiciones físicas para ser explorado por las raíces de los pastos. Los períodos de descanso han favorecido la estabilidad estructural del suelo y con ello la calidad del mismo. Se recomienda implementar prácticas tendientes a reducir los riesgos por compactación, que ayuden a frenar la degradación y a recuperar la capacidad productiva de estos suelos.

Palabras clave: porosidad, conductividad hidráulica, labranza, compactación

\section{Abstract}

The intensity of use and management of soil usuaIly alters the behavior of its physical properties and accelerates its degradation. In order to measure the impact of such intensity, a sampling was conducted at the experimental station of the CIAT-Palmira, Valle del Cauca, Colombia. Six lots with different histories, were chosen from very intensive six-month use to use reduced with rest for more than 10 years, and soil samples were taken at five depths of profile to evaluate the following properties: hydraulic conductivity, permeability to air, total porosity, drainable porosity, compactness, residual porosity, organic matter and mechanical 
resistance to penetration. It was found that the use of machinery and work of muddy improved physical properties only in $15 \mathrm{~cm}$ superficial, but they have caused serious problems of compaction in the lower depths. The trampling of livestock has also compressed profile, limiting the physical conditions to be explored by the grass roots. Rest periods have encouraged structural stability of soil and thus the quality of the same. It is recommended to implement practices to reduce risks due to compaction, to help curb the degradation and to restore the productive capacity of these soils

Key-words: porosity, hydraulic conductivity, tillage, compaction

\section{Resumo}

A intensidade de uso e manejo do solo geralmente altera o comportamento das suas propriedades físicas e acelera a sua degradação. A fim de medir o impacto de tal intensidade, que realizou uma estação experimental de amostragem CIATPalmira, Valle del Cauca, Colômbia. seis lotes com diferentes histórias foram escolhidas do uso semi muito intenso até reduzir para descansar por mais de 10 anos de uso, e amostras de solo foram coletadas em cinco profundidades Perfil para avaliar as seguintes propriedades: condutividade hidráulica, permeabilidade ao ar, porosidade total , porosidade drenável, densidade, porosidade residual, matéria orgânica e resistência mecânica à penetração. Verificou-se que o uso de maquinaria e o trabalho de pudlagem propriedades físicas melhoradas apenas na superfície de $15 \mathrm{~cm}$, mas causaram sérios compactação nas zonas mais baixas. Trampolim também compactado perfil, limitando a física para ser explorado pelas raízes das condições de pasto. Os períodos de descanso têm favorecido a estabilidade estrutural do solo e, portanto, a qualidade. Recomenda-se a implementação de práticas para reduzir os riscos de compactação, para ajudar a parar a degradação e restaurar a capacidade produtiva destes solos.

Palavras-chave: porosidade, condutividade hidráulica, preparo do solo, compactação

\section{Introducción}

Los suelos del Valle del Cauca, Colombia, se caracterizan por poseer una alta fertilidad natural que junto al relieve plano y el clima cálido han permitido en esta zona el desarrollo de un $60 \%$ de la agroindustria azucarera (Carbonell et al., 2001). No obstante, según varios autores citados por Madero et al. (2011), el uso intensivo de estos suelos y las constantes prácticas de laboreo bajo condiciones de alta humedad ocasionan problemas de degradación física reflejados en la estabilidad estructural, la organización del espacio poroso y la densidad en la capa arable, características que pueden limitar en las plantas la utilización de agua y nutrientes del suelo, también originar cambios en el régimen de humedad, la fertilidad y la eficiencia del riego.
La susceptibilidad de estos suelos a la degradación se debe a que el incremento en la intensidad de las labores de labranza convencional, expone la materia orgánica a una mayor descomposición, rompe los agregados del suelo (Lal, 1976) y disminuye su macroporosidad (Wagger \& Denton, 1989). Una reducción del carbono orgánico (CO) por debajo de los limites críticos además de acelerar la degradación de la estructura del suelo, incrementa la densidad aparente, disminuye la agregación y la capacidad de retención de humedad (Fageria, 2012) e impacta negativamente la producción de los cultivos (Fuentes et al., 2009).

Una forma de evitar la reducción del $\mathrm{CO}$ es realizar labores de cultivo menos intensivas mediante la 
rotación de cultivos (West \& Post, 2002) o el uso de no-labranza; esta última practica por ejemplo, restaura la estructura del suelos tropicales e incrementa el CO a una tasa de $0,43 \mathrm{Mg} / \mathrm{ha}$ por año en los 10 cm superficiales (Six et al., 2002). Según Wagger \& Denton (1989), un tráfico controlado es importante además para favorecer el estado físico general del suelo, el cual de acuerdo con IGAC (1990), comprende las condiciones de aire, agua y consistencia que influyen en el desarrollo de los cultivos.

Dados los efectos del uso y manejo intensivo sobre el suelo, se considera necesario evaluar cuantitativamente los impactos que producen las diferentes labores (Seben Junior, 2014), ya que éstos varían con el tipo de suelo y de cultivo (IGAC, 1990). Así, la presente investigación buscó evaluar los cambios físicos producidos por diferentes intensidades de uso y manejo en un suelo del Valle del Cauca, Colombia.

\section{Materiales y métodos}

El presente estudio se realizó en algunos lotes de cultivo de la sede principal del Centro Internacional de Agricultura Tropical - CIAT, localizado en el municipio de Palmira, departamento del Valle del Cauca, Colombia, a $3^{\circ} 30^{\prime}$ latitud norte, $76^{\circ} 19$, longitud oeste y una altitud de $965 \mathrm{msnm}$. Entre las condiciones climáticas, presenta una precipitación histórica promedio anual de $970 \mathrm{~mm}$, con un pico en los meses de marzo-mayo y otro en los meses de septiembre-noviembre, una temperatura promedio de $24^{\circ} \mathrm{C}$ y una humedad relativa de $74 \%$. Los suelos en su mayoría corresponden a Mollisoles.

Se seleccionaron seis lotes con historiales de más de 20 años bajo diferentes usos y manejo de los suelos como se describe la Tabla 1.

Tabla 1. Historial de uso de los lotes de muestreo en los últimos años.

\begin{tabular}{|c|c|c|}
\hline $\begin{array}{l}\text { Lote } \\
\text { CIAT }\end{array}$ & $\begin{array}{l}\text { Cultivo } \\
\text { Presente }\end{array}$ & Uso del Suelo \\
\hline $\mathrm{H}-3$ & Arroz & Nivelación, rastra y fangueo semestrales durante los últimos 28 años \\
\hline O-1 & Yuca & $\begin{array}{l}11 \text { años bajo subsolado, nivelado y cultivado con arroz, seguidos por } 10 \\
\text { años bajo subsolado cada dos años intercalado con pases de rastra y } \\
\text { establecido con una rotación Yuca, Maíz, Frijol, Sorgo. }\end{array}$ \\
\hline $\mathrm{R}-4$ & Caña & $\begin{array}{l}\text { Inicialmente potrero; luego, } 15 \text { años bajo una rotación Sorgo, Maíz, Yuca } \\
\text { seguidos por } 5 \text { años cultivado con Caña, subsolado anual, labores de } \\
\text { cultivo y cosecha mecanizadas. }\end{array}$ \\
\hline M-3 & Potrero & Suelo sin mecanización bajo pastoreo durante los últimos 40 años \\
\hline Q-3 & Frutales & $\begin{array}{l}20 \text { años bajo frutales, guadañado frecuentemente con tractor por } 10 \text { años } \\
\text { pero muy pocas veces los últimos } 10 \text { años. }\end{array}$ \\
\hline
\end{tabular}

En cada lote se escogieron al azar tres puntos de muestreo (calicatas), y se tomaron dos muestras de suelo (sin disturbar y disturbada) para cada una de las siguientes profundidades: 0-5, 5-10, 10-15, $15-20$ y $20-30 \mathrm{~cm}$. Las muestras fueron llevadas al Laboratorio de Física de Suelos del CIAT, donde a partir de la muestra en cilindros de $5 \times 5 \mathrm{~cm}$ se determinó conductividad hidráulica, permeabilidad al aire, humedad volumétrica a $75 \mathrm{~cm}$ de succión y densidad aparente, y con la muestra en bolsa plástica se determinó densidad real y materia orgánica. A partir de las dos densidades se calculó la porosidad total.

En el muestreo por cada profundidad, también, se incluyó una lectura con el Torvane para medir resistencia tangencial al corte y cuatro lecturas alrededor de cada calicata con un penetrógrafo DAIKI para 
medir la resistencia a la penetración. Después de llevar las muestras de los cilindros a $75 \mathrm{~cm}$ de succión, fueron sometidas a una presión de compactación de $33 \mathrm{PSI}$, para medir compactabilidad, porosidad residual y de nuevo su permeabilidad al aire.

Para cada variable se estimaron los promedios y desviaciones y se realizó un análisis de varianza al $5 \%$. El análisis estadístico de los datos incluyó también una comparación de medias utilizando la prueba Tukey.

\section{Resultados y discusión}

\section{Conductividad hidráulica y permeabilidad del aire}

El comportamiento de la conductividad hidráulica a través del perfil del suelo, en todos los lotes se presenta en la Figura 1. Se observa una menor variación en los lotes establecidos con caña y potrero, en los cuales se registraron las conductividades más bajas, asociadas con la compactación producida por el pisoteo del ganado hasta antes de sembrar la caña y durante los últimos años en el potrero. Los lotes con yuca y arroz, presentaron los valores más altos debido a la reciente preparación del suelo al momento del muestreo y se observó una disminución drástica en la conductividad a partir de los 15 $\mathrm{cm}$, indicando un efecto mínimo de la maquinaria debido a posibles pisos de arado. Aunque el lote con frutales exhibió también alta variabilidad en el perfil, mostró altas conductividades asociadas con gran presencia de raíces debido a una mayor estabilidad estructural alcanzada en ausencia de labranza.

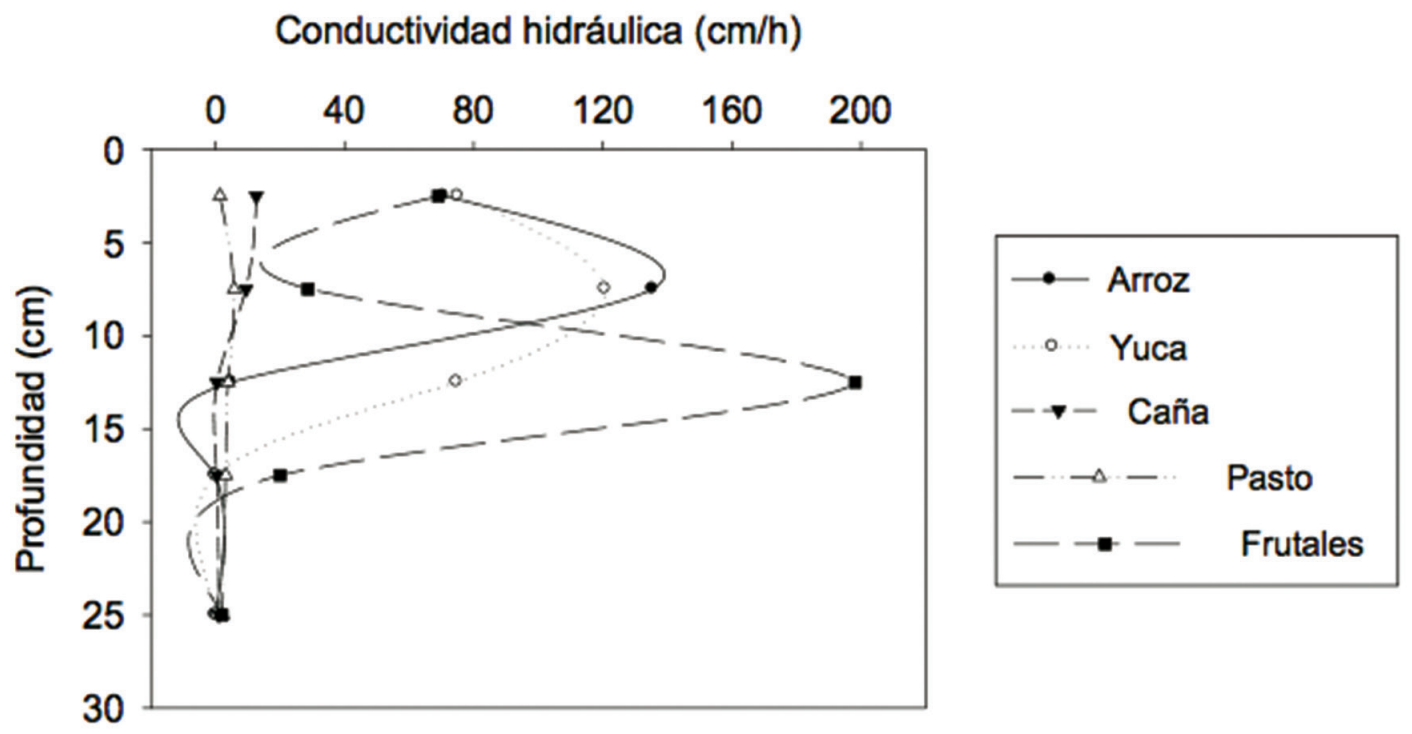

Figura 1. Comportamiento de la conductividad hidráulica en función del uso y manejo del suelo

En un estudio en Brasil, Soares et al. (2005) reportan que en suelos cultivados con caña de azúcar, el uso intensivo de maquinaria redujo la capacidad de infiltración superficial y el tamaño de los agregados, incrementando la relación micro/macro poros, mientras que las mayores conductividades se encontraron en sitios con estructura más estable por un alto contenido de arcillas.
Con relación a la permeabilidad del aire, los valores encontrados tanto antes como después de la compactación se presentan en la Figura 2. Similar a lo encontrado con la conductividad, las permeabilidades más bajas y una menor variabilidad se presentaron en los lotes con caña y potrero, indicando adensamiento o posible compactación en el perfil estudiado, mientras que los valores más 
altos se registraron en la superficie de los lotes recién preparados como resultado de la mecanización, así como en el lote con frutales en ausencia de labranza.

La Figura 2 también muestra que la permeabilidad es una propiedad muy sensible a la compactación, principalmente en los primeros centímetros de profundidad del perfil, reduciéndose hasta más de 20 veces en el suelo recién mecanizado del lote con yuca. En el lote con frutales se presentó la mayor reducción de la permeabilidad, como resultado de una mayor reducción de la macroporosidad, mientras que los lotes en potrero y caña mostraron las menores disminuciones, indicando un grado de alta compactación.
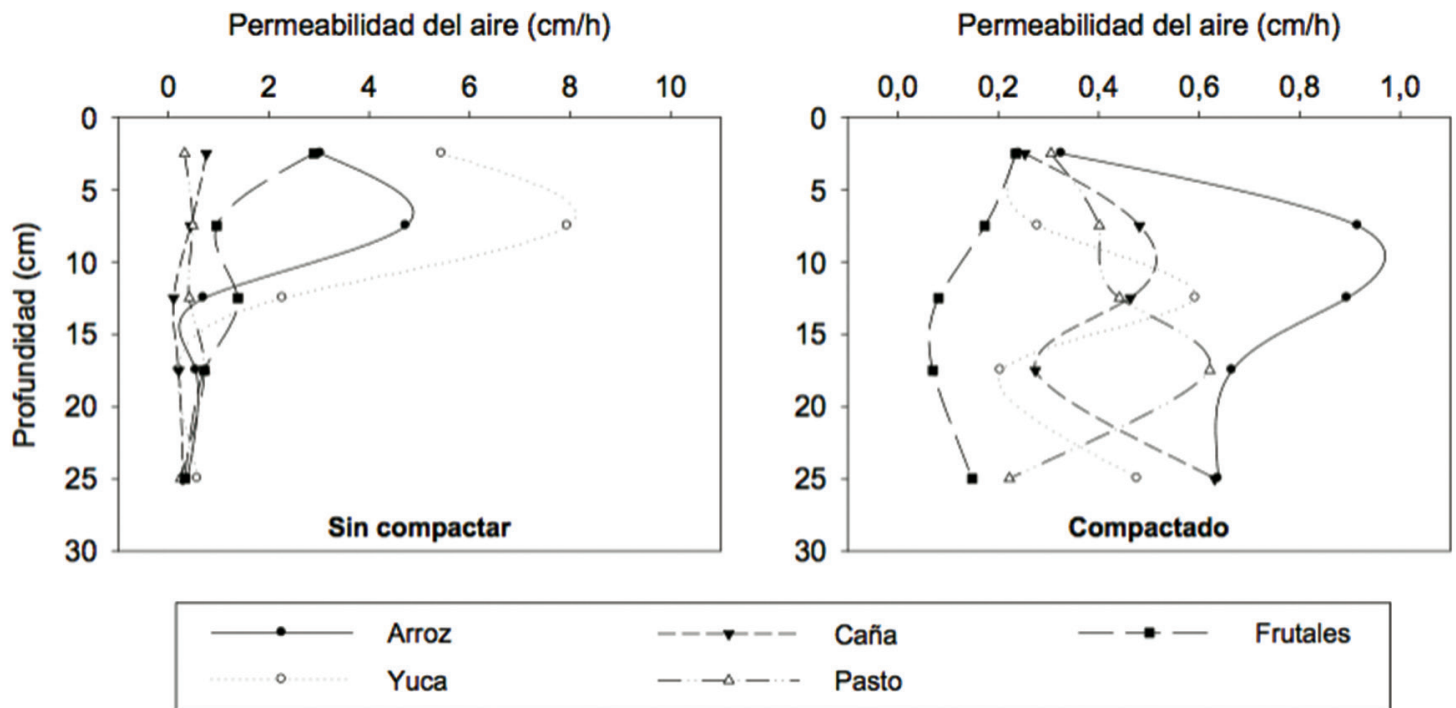

Figura 2. Comportamiento de la permeabilidad del aire (antes y después de la compactación) en función del uso y manejo del suelo

En la Tabla 2 se observa la relación entre la permeabilidad antes y después de la compactación. Los valores más altos indican un mayor efecto negativo de la compactación, y por ende una mayor susceptibilidad del suelo a la mecanización, mientras que los valores menores de 1, indican que, por el contrario, la permeabilidad se aumentó debido a que las muestras comenzaron a contraerse dejando grietas que permitieron un mayor flujo de aire.

Tabla 2. Relación entre la permeabilidad del aire antes/después de la compactación

\begin{tabular}{lllllc}
\hline Prof. $(\mathbf{c m})$ & Arroz & Yuca & Caña & Pasto & Frutales \\
\hline $0-5$ & 9.311 & 22.595 & 3.002 & 1.056 & 12.332 \\
$5-10$ & 5.169 & 28.499 & 0.929 & 1.218 & 5.555 \\
$10-15$ & 0.793 & 3.850 & 0.228 & 0.947 & 17.079 \\
$15-20$ & 0.835 & 1.068 & 0.770 & 1.165 & 10.279 \\
$20-30$ & 0.598 & 1.229 & 0.467 & 1.120 & 2.232 \\
\hline
\end{tabular}




\section{Porosidad total y porosidad drenable (poros > $40 \mathrm{~mm}$ )}

El comportamiento de las porosidades total y drenable (macroporos) se presenta en la Figura 3. Las dos propiedades exhibieron mayores valores en la superficie y disminuyeron al profundizar en el perfil de todos los lotes. Las mayores porosidades se registraron en los lotes con yuca, arroz y frutales, mientras que en los lotes con caña y pasto los menores valores indican un mayor adensamiento, especialmente a nivel subsuperficial.

Teniendo en cuenta que un valor de porosidad drenable $<10 \%$ puede ser considerada limitante para la buena aireación de las raíces, se observa que esta propiedad presentó valores restrictivos en los estratos inferiores de todos los perfiles, lo cual refleja un efecto marcado tanto de la preparación del suelo como de las raíces de las plantas, además de la flora y fauna sobre los macroporos, los cuales predominaron hacia la superficie donde también se registraron mayores contenidos de materia orgánica. En los lotes con arroz y yuca, el uso de maquinaria ha reducido en mayor proporción la macroporosidad, mientras que en el lote con frutales, los valores altos se asocian con la ganancia de estabilidad estructural en ausencia de mecanización. En el lote en potrero, donde casi todo el perfil sería limitante, se refleja de nuevo el efecto adverso que tiene el pisoteo del ganado en las propiedades físicas del suelo.

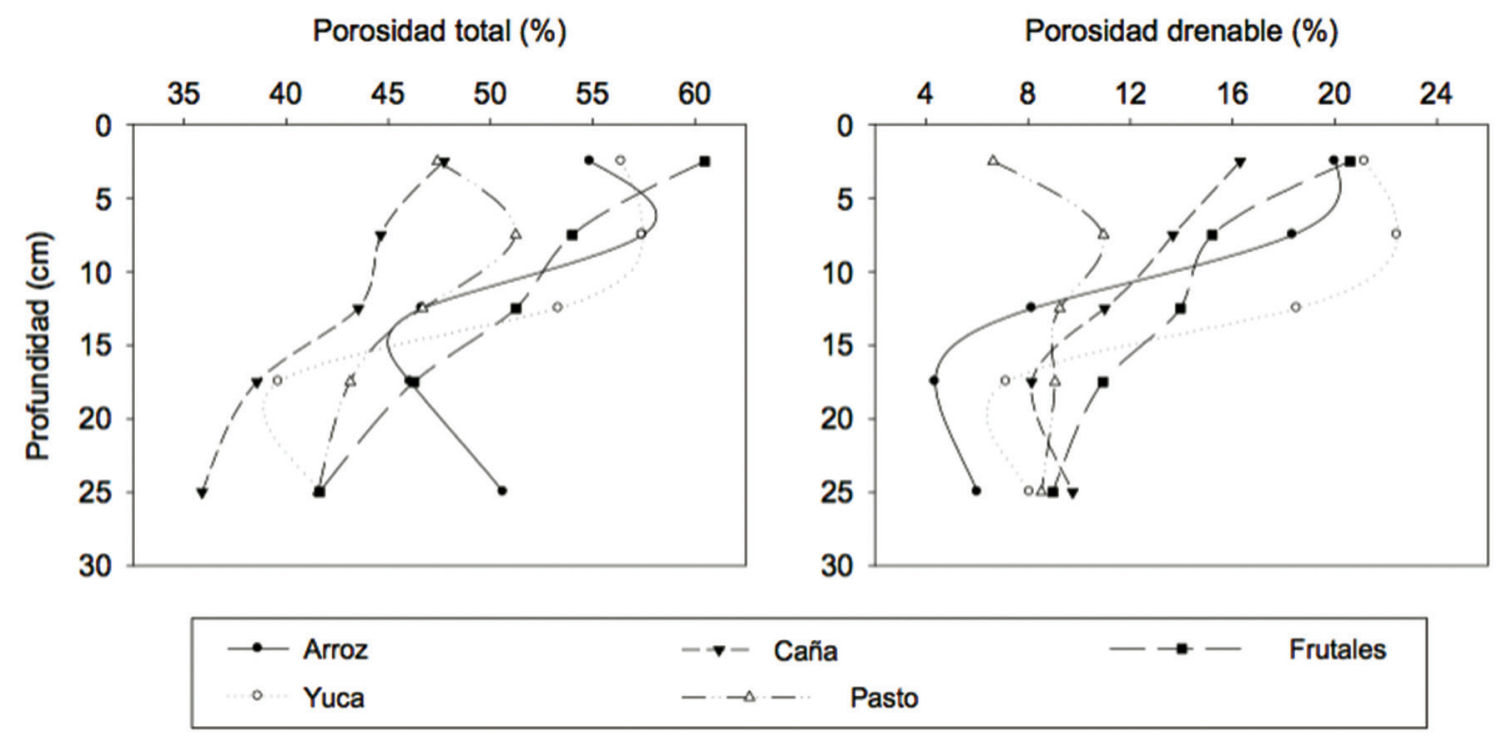

Figura 3. Comportamiento de la porosidad total y drenable del suelo en función del uso y manejo

Similarmente, Volverás \& Amézquita (2009), al comparar dos suelos con y sin uso agrícola, reportan que el cambio de uso del suelo afecta negativamente su volumen aún en periodos cortos y que la porosidad total tiende a disminuir al aumentar el tiempo de uso hasta incluso 70 años de uso agrícola. En otro estudio, Lanzanova et al. (2010) reportan que después de 16 años de utilizar la prácticas como no labranza, rotación de cultivos y cultivos de cobertura se incrementa la porosidad y la macroporosidad del suelo en comparación con un suelo desprotegido.

\section{Compactabilidad y Porosidad residual}

En la Figura 4 se presenta el comportamiento de la compactabilidad y de la porosidad residual. 
Similar a las propiedades anteriores, los perfiles de los lotes sembrados con arroz y yuca exhibieron los menores valores de compactabilidad en la superficie como resultado de la preparación del suelo, mientras que los mismos lotes en los niveles subsuperficiales junto con los lotes establecidos con caña y potrero presentaron las mayores compactibilidades, producto del uso intensivo que ha llevado a la formación de pisos de arado. No obstante, el lote con frutales mostró menor compactación en los niveles subsuperficiales debido a la acción de las raíces.
Respecto a la porosidad residual, esta propiedad exhibió un comportamiento inverso a la compactabilidad; los valores altos que indican mayor espacio para explorar por las raíces se incrementaron en los lotes con labranza reciente, al igual que en el lote con frutales en los primeros centímetros de profundidad, indicando que la ausencia de labores culturales en el suelo tiende a recuperar la porosidad residual del suelo, mientras que el lote bajo potrero siguió mostrando el efecto adverso causado por la carga animal.

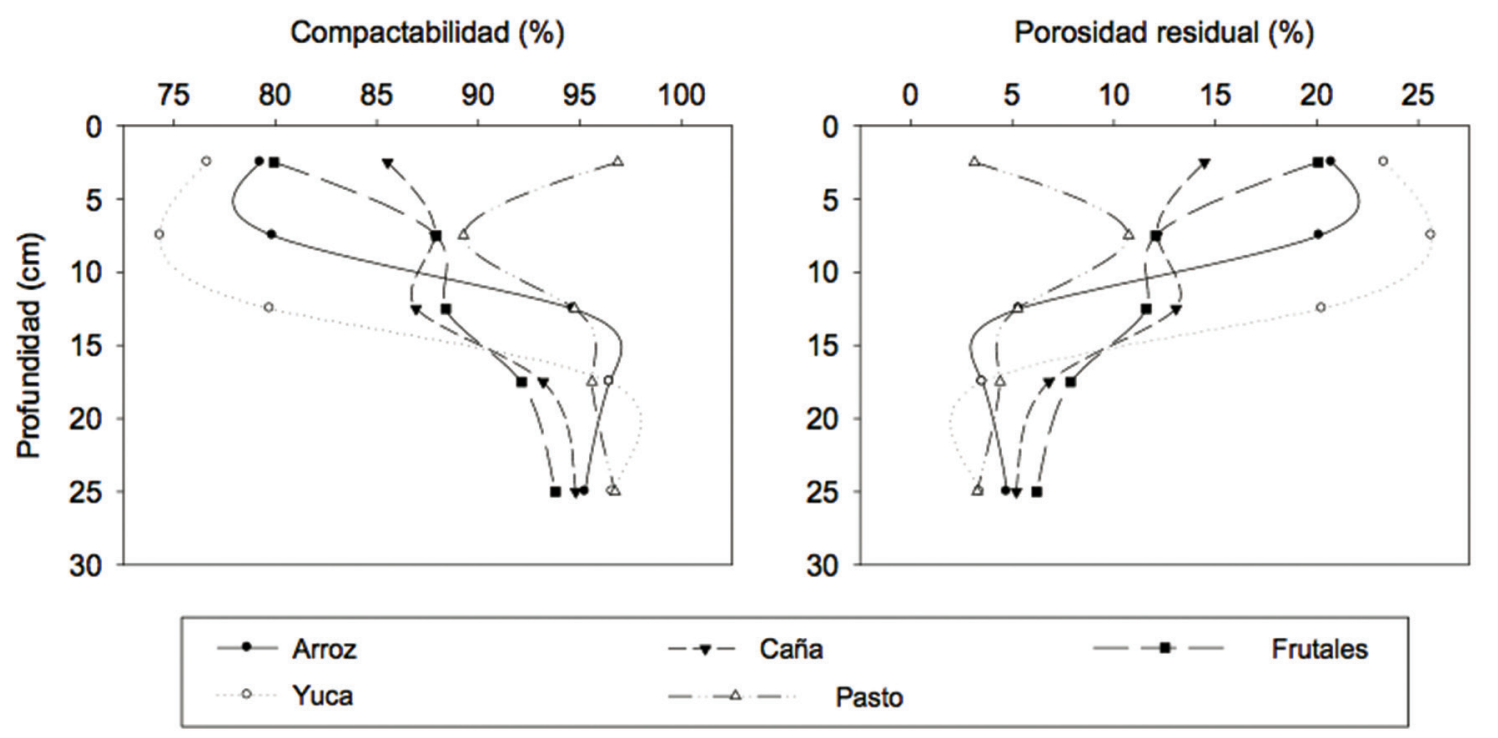

Figura 4. Comportamiento de la compactibilidad y la porosidad residual en función del uso y manejo del suelo

\section{Materia orgánica}

Como se observa en la Figura 5, los contenidos de materia orgánica para los diferentes lotes muestran una tendencia a disminuir al profundizar en todos los perfiles, sobresaliendo los lotes en pastoreo y frutales con los valores más altos, como señal de que la compactación del suelo y la poca intervención antrópica disminuyen las tasas de mineralización de la materia orgánica.
El lote con arroz evidencia que el fangueo del suelo influye en la redistribución de la materia orgánica a través del perfil y también disminuye su mineralización. Los valores bajos en el lote con caña reflejan el agotamiento de materia orgánica debido a la alta extracción del cultivo y posiblemente a la quema de residuos a la cual estos lotes fueron expuestos anteriormente. 


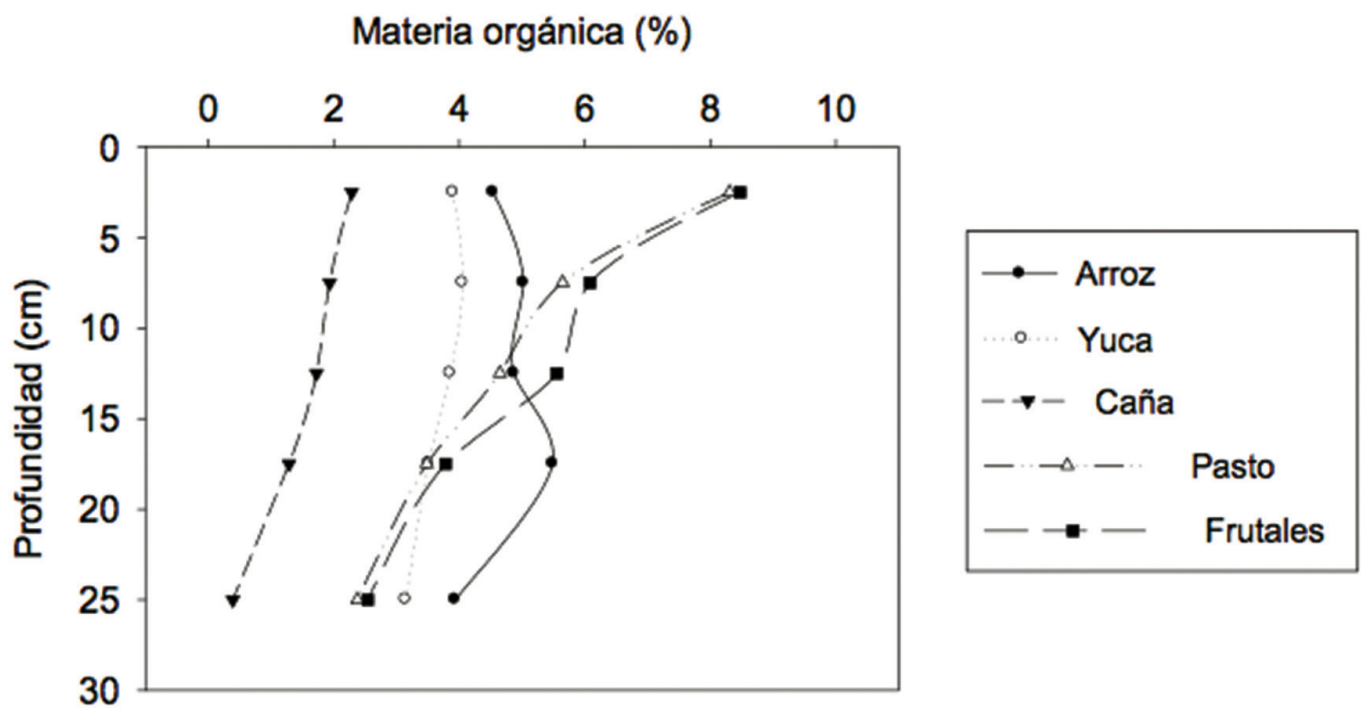

Figura 5. Comportamiento de la materia orgánica en función del uso y manejo del suelo

\section{Resistencia del suelo al corte y a la penetración}

El efecto de la reciente preparación del suelo en los lotes con yuca y arroz, con valores de resistencia tangencial al corte bajos, así como el efecto de la compactación del suelo causada por el pisoteo del ganado reflejado en valores muy altos a través de todo el perfil se presenta en la Figura 6. En los lotes con caña y frutales, a pesar de mostrar menor resistencia en la superficie, muestran una tendencia a aumentar ésta al profundizar el perfil, como en todos los lotes asociados con adensamientos o pisos de arado en los niveles subsuperficiales del suelo causados por la labranza.

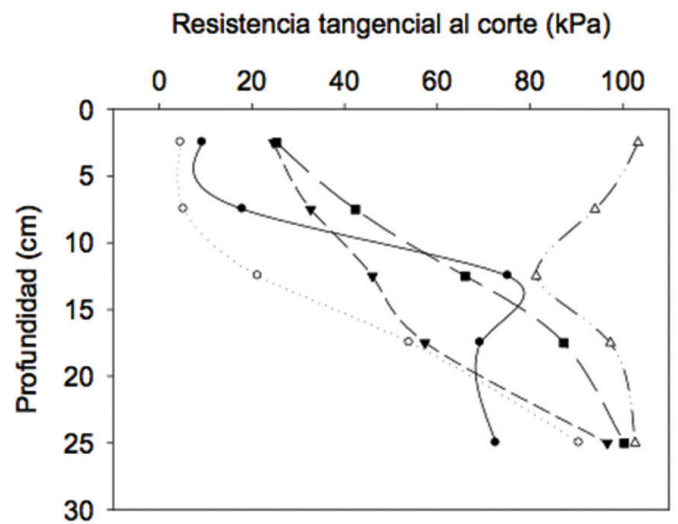

La resistencia a la penetración evidencia también en los primeros centímetros de profundidad de los perfiles el efecto marcado de la reciente preparación en los lotes con arroz y yuca y del pisoteo del ganado en el potrero (Figura 6).

Similar a lo observado en el lote con frutales donde se redujo la resistencia del suelo en la superficie, Seben Junior et al. (2014) afirman que este efecto de la no labranza se asocia con la formación de bioporos, los cuales son requeridos para mantener la función porosa del suelo y de sus propiedades mecánicas a través del tiempo.

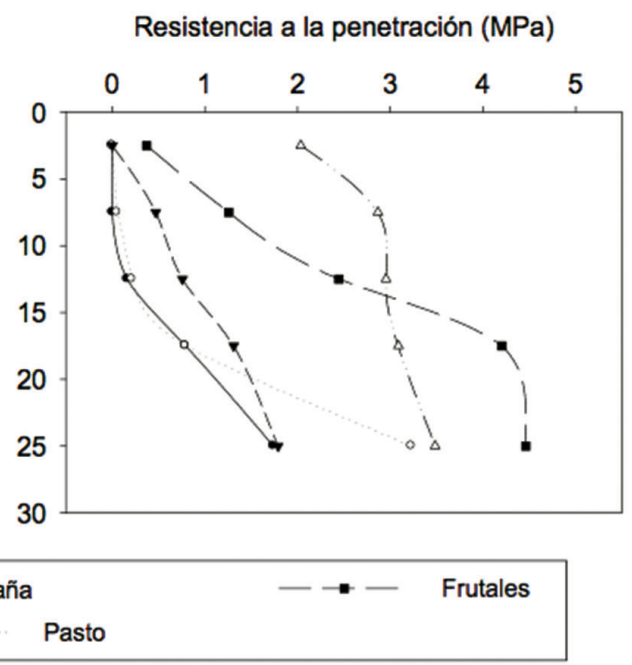

Figura 6. Comportamiento de la resistencia del suelo en función del uso y manejo 


\section{Conclusiones}

La reciente preparación del suelo causa un efecto positivo en las condiciones físicas del suelo sólo en los primeros 15 centímetros de profundidad de los lotes, pero su uso continuado a través del tiempo ha causado pisos de arado o compactación a nivel subsuperficial.

Largos periodos de descanso y la ausencia de labranza en el lote con frutales ha favorecido las propiedades físicas, pero esta tasa de recuperación es mucho menor que los efectos adversos del uso intensivo del suelo y el pisoteo del ganado.

Es necesario, entonces, implementar prácticas de manejo que ayuden a disminuir la compactación del perfil y por ende la degradación del suelo, y a recuperar la capacidad productiva de los suelos tropicales.

\section{Agradecimientos}

Los autores agradecen a todas las personas que colaboraron con realización de este estudio, especialmente a Arvey Álvarez y Jesús Galvis quienes ayudaron en la toma y análisis de las muestras en el laboratorio de Física de Suelos del CIAT.

\section{Literatura citada}

1. Carbonell, J., Amaya, A., Ortiz, B., Torres, J., Quintero, R. \& Isaacs, C. (2001). Zonificación agroecológica para el cultivo de caña de azúcar en el valle del río Cauca, tercera aproximación. Cali, Centro de Investigación de la Caña de Azúcar de Colombia (Cenicaña). (Serie Técnica No 29).

2. Fageria, N.K. (2009). Role of soil organic matter in maintaining sustainability of cropping systems. Communications in Soil Science and Plant Analysis 43:2063-2113. 2012.
3. Instituto Geografico Agustín Codazzi (IGAC). (1990). Propiedades físicas de los suelos. Bogotá, Subdirección Agrológica. $813 p$

4. Lal, R. (1976). No-tillage effects on soil properties under different crops in Western Nigeria. Soil Science Society of America Journal 40:762-768.

5. Lanzanova, M.E., Eltz, F.L.F., Nicoloso, R.S., Amado, T.J.C., Reinert, D.J. \& Rocha, M.R. (2010). Atributos físicos de um Argissolo em sistemas de culturas de longa duração sob semeadura direta. Revista Brasileira de Ciência do Solo, 34:1333-1342.

6. Madero Morales, E., Ramírez Alzate, J.A., Albán, Á., Escobar, B.Y., García, L.F. \& Peña Artunduaga, M.E. (2011). Compactación de suelos cultivados con caña de azúcar en la zona sur del Valle del Cauca. Parte I. Acta Agronómica 60 (3): 245-252.

7. Seben Junior, G. De F., Cora, J.E. \& Lal, R. (2014). The effects of land use and soil management on the physical properties of an Oxisol in Southeast Brazil. Revista Brasileira de Ciência do Solo 38(4): 1245-1255. Doi: 10.1590/S0100-06832014000400021.

8. Six, J., Feller, C., Denef, K., Ogle, S.M., Sá, J.C.M. \& Albrecht, A. (2002). Soil organic matter, biota and aggregation in temperate and tropical soils - Effects of notillage. Agronomie 22:755-775.

9. Soares, J.L.N., Espindola, C.R. \& Pereira, W.L.M. (2005). Physical properties of soils under intensive agricultural management. Scientia Agricola 62(2): 165-172.

10. Volverás, B. \& Amézquita, E. (2009). Estabilidad estructural del suelo bajo diferentes sistemas y tiempo de uso en laderas andinas de Nariño, Colombia. Acta Agronómica 58(1): 35-40.

11. Wagger, M.G. \& Denton, H.P. (1989). Influence of cover crop and wheel traffic on soil physical properties in continuous no-till corn. Soil Science Society of America Journal 53(4): 1206-1210. Doi: 10.2136/ sssaj1989.03615995005300040036x

12. West, T.O. \& Post, W.M. (2002). Soil organic carbon sequestration rates by tillage and crop rotation: A global data analysis. Soil Science Society of America Journal 66:1930-1946.

\section{Conflicto de Intereses}

Los autores declaran no tener ningún conflicto de intereses 
